\title{
Obesity is associated with hypothalamic injury in rodents and humans
}

\author{
Joshua P. Thaler, ${ }^{1,2}$ Chun-Xia Yi, ${ }^{3}$ Ellen A. Schur, ${ }^{2}$ Stephan J. Guyenet, ${ }^{1,2}$ Bang H. Hwang,, ${ }^{1,2,4}$ \\ Marcelo O. Dietrich, ${ }^{5}$ Xiaolin Zhao, ${ }^{1,2,6}$ David A. Sarruf,,1,2 Vitaly Izgur, ${ }^{7}$ \\ Kenneth R. Maravilla,7 Hong T. Nguyen,1,2 Jonathan D. Fischer, ${ }^{1,2}$ Miles E. Matsen,,1,2 \\ Brent E. Wisse,1,2 Gregory J. Morton,1,2 Tamas L. Horvath,5,8 Denis G. Baskin,1,2,4 \\ Matthias H. Tschöp, ${ }^{3}$ and Michael W. Schwartz',2

\begin{abstract}
${ }^{1}$ Division of Metabolism, Endocrinology and Nutrition, Diabetes and Obesity Center of Excellence, and 2Department of Medicine, University of Washington, Seattle, Washington, USA. ${ }^{3}$ Metabolic Diseases Institute, Division of Endocrinology, Department of Medicine, University of Cincinnati, Cincinnati, Ohio, USA. ${ }^{4}$ Research and Development Service, Department of Veterans Affairs Puget Sound Health Care System, Seattle, Washington, USA. ${ }^{5}$ Program in Integrative Cell Signaling and Neurobiology of Metabolism, Section of Comparative Medicine, Yale University School of Medicine, New Haven, Connecticut, USA. ${ }^{6}$ Department of Physiology and Pathophysiology,

School of Medicine at Xi'an Jiaotong University, Xi'an, China. ${ }^{7}$ Department of Radiology, University of Washington, Seattle, Washington, USA.
\end{abstract} \\ ${ }^{8}$ Department of Obstetrics/Gynecology and Reproductive Sciences, Yale University School of Medicine, New Haven, Connecticut, USA.
}

\begin{abstract}
Rodent models of obesity induced by consuming high-fat diet (HFD) are characterized by inflammation both in peripheral tissues and in hypothalamic areas critical for energy homeostasis. Here we report that unlike inflammation in peripheral tissues, which develops as a consequence of obesity, hypothalamic inflammatory signaling was evident in both rats and mice within 1 to 3 days of HFD onset, prior to substantial weight gain. Furthermore, both reactive gliosis and markers suggestive of neuron injury were evident in the hypothalamic arcuate nucleus of rats and mice within the first week of HFD feeding. Although these responses temporarily subsided, suggesting that neuroprotective mechanisms may initially limit the damage, with continued HFD feeding, inflammation and gliosis returned permanently to the mediobasal hypothalamus. Consistent with these data in rodents, we found evidence of increased gliosis in the mediobasal hypothalamus of obese humans, as assessed by MRI. These findings collectively suggest that, in both humans and rodent models, obesity is associated with neuronal injury in a brain area crucial for body weight control.
\end{abstract}

\section{Introduction}

Obesity has emerged as a major health problem in industrialized nations. Despite substantial progress in understanding the neurobiology of energy homeostasis (the biological process through which energy intake and expenditure are matched to one another so as to promote stability in the amount of fuel stored as fat) (1), little is known regarding how brain systems designed to promote weight stability are altered in common forms of obesity $(2,3)$.

Growing evidence implicates immune cell-mediated tissue inflammation as an important mechanism linking obesity to insulin resistance in metabolically active organs, such as liver, skeletal muscle, and adipose tissue (4-6). In rodent models of diet-induced obesity (DIO), increased inflammatory signaling in the mediobasal hypothalamus $(\mathrm{MBH})$ similarly contributes to leptin resistance and weight gain (7-12), but the cellular interactions underlying this inflammatory response remain uncharacterized. The goal of the current study was to identify the neuroanatomical correlates of obesity-associated hypothalamic inflammation and to determine whether similar responses occur in humans.

We report that unlike inflammation in peripheral tissues, a process that develops over weeks to months of high-fat diet (HFD) feeding in rodent models (13-15), markers of hypothalamic inflammation are elevated within 24 hours of HFD exposure. Within the first week of HFD, markers of neuron injury also become evident in the hypothalamic arcuate nucleus (ARC) and

Authorship note: Chun-Xia Yi and Ellen A. Schur contributed equally to this work. Conflict of interest: The authors have declared that no conflict of interest exists. Citation for this article: J Clin Invest doi:10.1172/JCI59660. adjacent median eminence (ARC-ME) in association with reactive gliosis involving recruitment of both microglia and astrocytes. Although initially transient, suggesting an effective neuroprotective response, inflammation and gliosis return and become established with continued HFD exposure. Using an established MRI method (16-19), we also report evidence of increased gliosis in the $\mathrm{MBH}$ of obese humans. These findings collectively suggest that, in both humans and rodent models, obesity is associated with injury to a key brain area for energy homeostasis.

\section{Results}

Time course of HFD-induced hypothalamic inflammatory gene expression. Consistent with a large volume of literature $(7-11,13,14,20)$, we found that expression of several proinflammatory genes increased by approximately $50 \%$ in both hypothalamus (Figure $1 \mathrm{~A}$ ) and liver (Figure 1B) of adult male rats subjected to long-term (20 weeks) consumption of a HFD ( $60 \%$ of calories from fat) relative to that in controls fed standard chow. By comparison, whereas inflammation was not detected in either liver or adipose tissue after only 4 weeks of HFD, hypothalamic inflammation was clearly evident at this earlier time point (Figure 1, C-E). Thus, the effect of HFD feeding to induce hypothalamic inflammatory gene expression seems unlikely to arise from a systemic inflammatory process.

Since rats fed the HFD for 4 weeks gained more than $50 \mathrm{~g}$ more body weight and more than $10 \%$ more fat mass than chow-fed controls over the same time frame (data not shown), it remains possible that hypothalamic inflammation at this time point is a consequence of obesity. To address this question, we analyzed hypothalamic proinflammatory gene expression in rats during the 

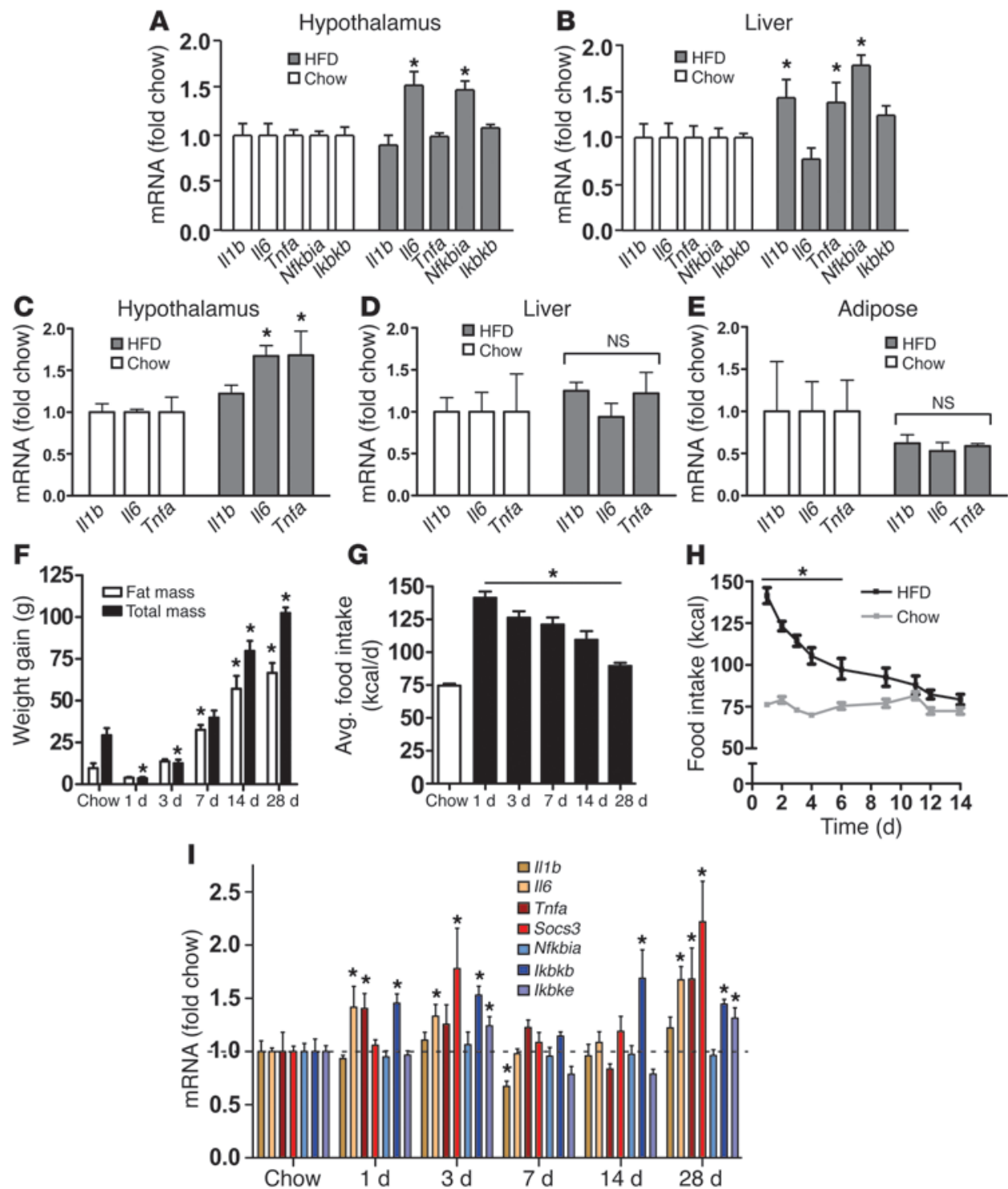

Figure 1

Time course of hypothalamic inflammation after the onset of HFD feeding. (A and B) Quantification of mRNA encoding proinflammatory cytokines $(I / 1 b, I / 6, T n f a)$ and NF-kB pathway genes (Nfkbia, lkbkb) in (A) hypothalamus and (B) liver of rats fed either standard chow (white bars) or HFD (gray bars) for 20 weeks ( $n=6 /$ group). ${ }^{*} P<0.05$ versus chow-fed controls. (C-E) Effect of 4 weeks of HFD feeding (gray bars) on proinflammatory cytokine gene expression in rat (C) hypothalamus, (D) liver, and (E) white adipose tissue compared with that in chow-fed controls (white bars) ( $n=6 /$ group). ${ }^{*} P<0.05$ versus chow-fed controls. (F) Total weight gain (black bars), fat mass gain (white bars), and (G) average (avg) daily food intake (kcal/d) of rats ( $n=6 /$ group) fed chow for 2 weeks or HFD for up to 28 days. ${ }^{*} P<0.05$ versus chow-fed controls. (H) Comparison of daily food intake (kcal) in rats $\left(n=6 /\right.$ group) fed chow (gray) or HFD (black) for 14 days. ${ }^{*} P<0.05$ versus chow-fed controls. (I) Time course of induction of mRNA encoding inflammatory mediators, including proinflammatory cytokine (II1 b, I/6, Tnfa), cytokine pathway (Socs3), and NF-kB pathway (Nfkbia, Ikbkb, Ikbke) gene expression in the hypothalamus of rats fed chow or HFD for up to 28 days $(n=6 / \mathrm{group})$. All mRNA species were quantified relative to $18 \mathrm{~S}$ and Gapdh housekeeping gene expression (by $\Delta \Delta \mathrm{CT}$ method) and presented as fold change relative to chow-fed controls [fold chow]. The dashed line in I represents the level of expression equal to chow-fed controls. ${ }^{*} P<0.05$ versus chow.

initial phase of HFD feeding. Relative to that of chow-fed controls, rats placed on the HFD exhibited a transient but robust $(50 \%-100 \%)$ increase of food intake (Figure $1, \mathrm{G}$ and $\mathrm{H}$ ) that gave rise to a small but significant increase of body weight and fat mass by day 7 (Figure $1 \mathrm{~F}$ ). In this cohort, gene expression analysis revealed a complex "on-off-on" pattern, with elevated hypothalamic levels of Il6, Tnfa, Socs $3, I k b k b$, and Ikbke mRNA observed within the first 3 days of HFD exposure, followed by a decline to baseline values from days
7 to 14 and a subsequent return to elevated levels by day 28 (Figure 1I). Thus, increases of hypothalamic proinflammatory gene expression closely mirrored changes of energy intake during the first days of HFD feeding, and both occurred prior to significant expansion of body fat mass ( $P=$ NS for fat mass gain of HFD-fed rats at days 1 or 3 compared with chow-fed controls; Figure $1 \mathrm{~F}$ ). C57BL/6 mice displayed a similar, transient increase of proinflammatory gene expression during the first 7 days of HFD feeding 

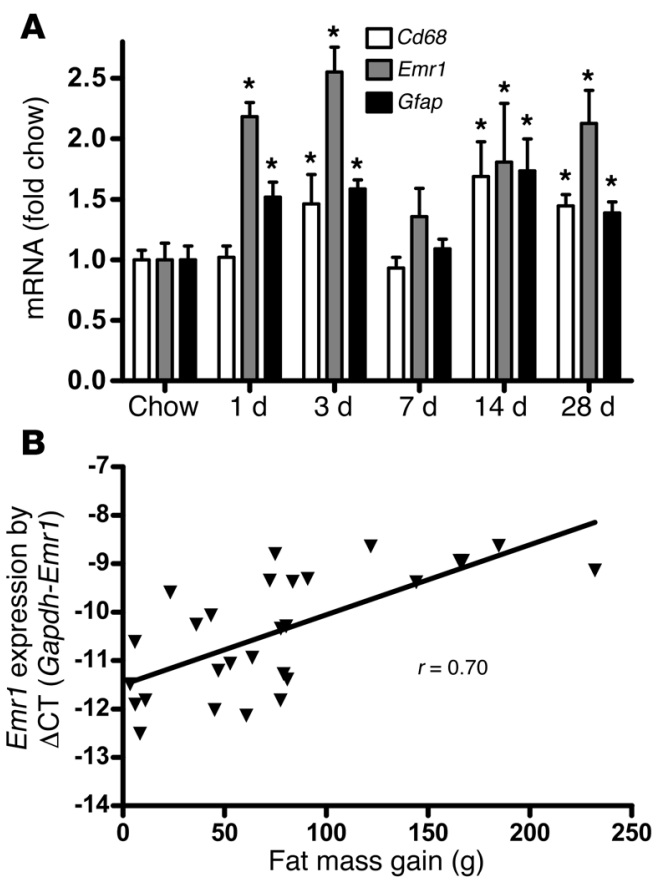

Figure 2

Effect of HFD feeding on hypothalamic microglial markers in rats. (A) Time course of hypothalamic microglia-specific (Cd68 and Emr1) and astrocyte-specific (Gfap) gene expression in rats fed chow or HFD for up to 28 days ( $n=6 /$ group). ${ }^{*} P<0.05$ versus chow. (B) Correlation of hypothalamic Emr1 mRNA level (linearized using difference in CT count between the Gapdh and the Emr1 gene [ $\Delta \mathrm{CT}]$ ) with change in fat mass $(\mathrm{g})$ over 4 weeks of HFD feeding $(r=0.70 ; P<0.001)$.

(Supplemental Figure 1; supplemental material available online with this article; doi:10.1172/JCI59660DS1), indicating that hypothalamic inflammatory signaling occurs prior to substantial weight gain in mice, as it does in rats.

Markers of gliosis during early HFD feeding. Activation, recruitment, and proliferation of microglia (macrophage-like immune cells of the brain) and astrocytes, collectively termed "reactive gliosis," are hallmarks of the brain response to neuronal injury (21-24). We observed that, in rats fed a HFD for up to 28 days, hypothalamic expression of mRNA encoding myeloid cell-specific markers $\mathrm{Cd} 68$ and Emr 1 (which encodes F4/80) increased by $50 \%-100 \%$ by day 3 (Figure 2A), suggesting an effect of HFD feeding to promote microglial accumulation in this brain area. Interestingly, the level of hypothalamic Emr1 gene expression was correlated with fat mass gain $(r=0.70 ; P<0.001$; Figure $2 \mathrm{~B})$. Combined with our finding that hypothalamic expression of mRNA encoding the astrocyte marker gene Gfap is comparably increased at the same early time point (Figure 2A), these results suggest that the rapid onset of hypothalamic inflammation induced by HFD feeding is associated with robust glial responses.

Using immunohistochemistry to detect the microglia-specific cytoplasmic marker Iba1 (ref. 25 and Figure 3A), we found that microglial number increased in rat ARC by day 3 of HFD exposure compared with that in chow-fed controls (Figure 3, B-D) and remained elevated throughout the initial 2-week period (Figure 3, $\mathrm{E}$ and F; quantified in Figure 3G). Concomitantly, microglial cells enlarged (Figure $3 \mathrm{H}$ ) and adopted a more activated morphology (compare Figure 3I with Figure 3J). In separate cohorts of rats fed a
HFD for up to 8 weeks, microglial accumulation in the ARC correlated with the degree of fat mass gain $(r=0.54, P=0.019$; Figure $3 \mathrm{~K})$, and animals with greater increases of fat mass also had larger microglia in the ARC $(r=0.52, P=0.028$; Figure $3 \mathrm{~L})$. Moreover, changes of both microglial accumulation and cell size appear to be limited to the ARC-ME region, as they were not observed in other hypothalamic (lateral hypothalamic area, ventromedial nucleus) or extrahypothalamic (hippocampus, cerebral cortex) brain areas (Supplemental Figure 2). Since an analogous accumulation of microglia occurred in the ARC-ME of wild-type C57BL/6 mice (Supplemental Figure 3), this effect of HFD feeding is not unique to rats.

The effect of HFD on astroglial responses was assessed in C57BL/ 6 mice by GFAP immunostaining. As expected, GFAP-positive astrocytes were scattered throughout the ARC-ME of mice fed standard chow, and their processes resembled those in other brain areas (Figure 4A). Within just 1 week of HFD exposure, however, the intensity of GFAP staining in the ARC-ME increased by approximately 50\% (Figure 4, B and G). Like hypothalamic inflammatory markers (and unlike the microglial response), this astrocytosis was transient, returning to baseline in mice fed the HFD for 2 to 3 weeks (Figure 4, C and D, respectively; quantified in Figure 4G) but recurring with long-term HFD feeding relative to that in agematched controls fed chow for the same duration (Figure 4, E and F; quantified in Figure 4H). A similar pattern of hypothalamic gliosis was observed in rats over the first 8 weeks of HFD feeding (data not shown), confirming that comparable microglial, astroglial, and inflammatory responses to HFD occur in both species.

Using immunohistochemistry to detect GFAP, we observed the expected preservation of distinct cell-cell boundaries among astrocytes in the ARC-ME of chow-fed mice (Figure 5A). After just 1 week of HFD exposure, however, astrocytic processes in this brain area coalesced into a dense fibrous network suggestive of a syncytium (Figure 5B), a finding pathognomonic of reactive gliosis (22). This effect was initially transient and resolved between weeks 2 and 3 of HFD (Figure 5, C and D) but reappeared in mice fed a HFD for long intervals (8 months; Figure 5, E and F).

HFD-induced neuronal injury. The rapid onset of inflammation and reactive gliosis observed in the hypothalamus of rats and mice consuming a HFD is a hallmark of the response to neuron injury (e.g., induced by ischemia or excitotoxicity) (21-24). To more directly test the hypothesis that HFD exposure causes ARC neuron injury, we performed immunohistochemical staining to detect induction of the chaperone Hsp72, a component of the neuroprotective response to neuron injury (26), in the ARC-ME of rats fed the HFD for 7 days relative to that of chow-fed controls (Figure 6, A and B). As predicted, Hsp72 immunostaining was increased in HFD-fed rats relative to that in chow-fed controls, and hypothalamic $H s p 72$ mRNA expression was also detectably elevated within 3 days of HFD exposure (data not shown). Among ARC neurons in which $\mathrm{Hsp} 72$ induction was detected are those containing proopiomelanocortin (POMC) that are components of a critical network for energy balance regulation (ref. 1 and Figure 6, C and D).

We next used electron microscopy to investigate whether autophagy, an independent marker of neuronal stress/injury $(27,28)$, was induced in POMC neurons from C57BL/ 6 mice fed the HFD (Figure 6, E-I). After 20 weeks of HFD feeding, the percentage of POMC neurons with detectable autophagosomes increased more than 10 fold (black arrows in Figure 6F; higher-magnification view in Figure 6G), such that the majority of POMC cells examined now exhibited this organelle $(6.4 \% \pm 4.1 \%$ in chow fed vs. $81.2 \% \pm 8.7 \%$ in HFD fed; 

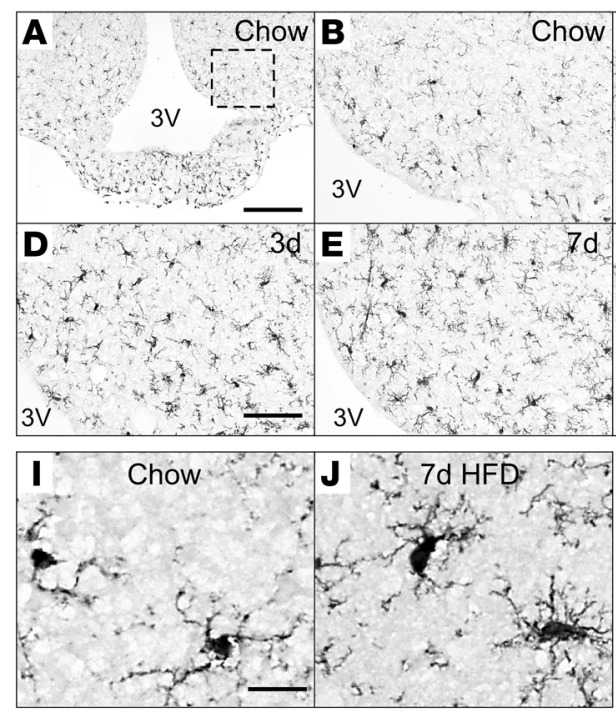

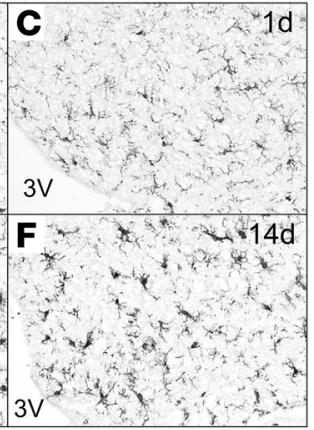

$\mathbf{K}$

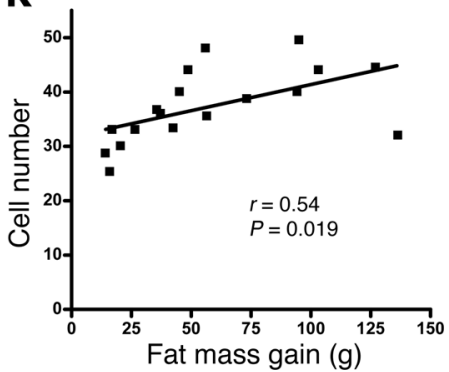

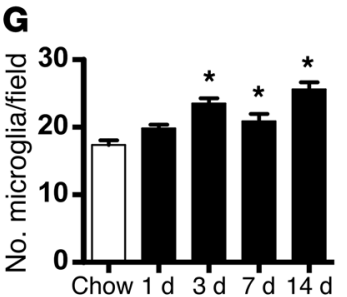

H

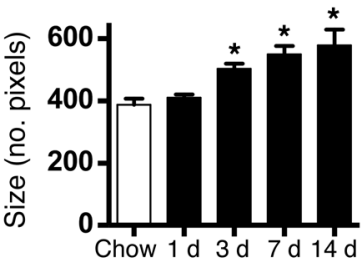

$\mathbf{L}$

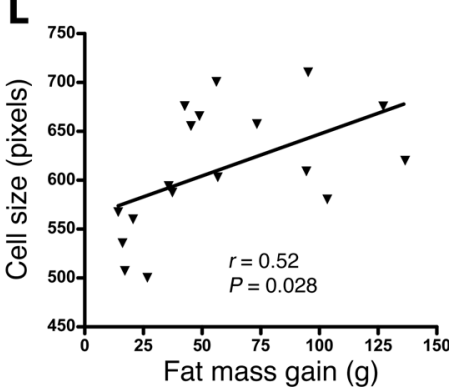

Figure 3

Histochemical analysis of HFD-induced microglial accumulation in rat ARC. Immunohistochemical detection of Iba1 protein, a microglial marker (25), in coronal sections of rat hypothalamus $(14 \mu \mathrm{m})$ from animals fed either (A and B) chow or (C-F) HFD for up to 14 days. (A) Low-magnification view (original magnification, $\times 10$ ) of microglia distributed throughout the $\mathrm{MBH}$. The dashed box indicates the region used for quantification of ARC microglial number and size in G and H. 3V, third ventricle. Scale bar: $100 \mu \mathrm{m}$. (B-F) Higher-magnification view (original magnification, $\times 20$ ) of Iba1 immunohistochemistry in the ARC of rats fed (B) chow or (C) HFD for 1 day, (D) 3 days, (E) 7 days, or (F) 14 days. Scale bar: 50 um. ( $\mathbf{G}$ and $\mathbf{H}$ ) Quantification of $(\mathbf{G})$ mean ARC microglial cell number (per field defined in $\mathbf{A}$ ) and $(\mathbf{H})$ microglial cell size (average number of pixels in 10 largest cells) from rats fed either chow or HFD ( $n=6 /$ group). ${ }^{*} P<0.05$ versus chow. (I and $\left.\mathbf{J}\right)$ Comparison of microglial fine structure in hypothalamus of rats fed (I) chow or (J) HFD for 7 days. Microglia from HFD-fed rats manifest a more "ameboid" morphology, characterized by larger cell bodies with thickened and shortened processes. Scale bar: $10 \mu \mathrm{m}$. (K) Correlation of microglial cell number and fat mass gain ( $\mathrm{g}$ ) over 2-8 weeks of HFD consumption, with indicated linear regression line. (L) Correlation of microglial cell size (no. pixels in 10 largest cells/ARC) and fat mass gain $(\mathrm{g})$ over 2 to 8 weeks of HFD consumption, with indicated linear regression line. Each symbol in $\mathbf{K}$ and $\mathbf{L}$ represents an animal.

$P=0.00005 ;$ Figure 6I). In addition, a disruption of mitochondrial morphology was observed in POMC neurons from mice fed a HFD (white arrows in Figure 6, E and F; higher-magnification view in $6 \mathrm{H})$. Whereas mitochondria in POMC neurons from chow-fed rats uniformly demonstrated homogenous, compact electron-dense lumens with well-organized, parallel-oriented cristae (Figure 6H), mitochondria from HFD-fed animals manifested irregular swellings and less electron-dense lumen with nonparallel cristae that were frequently discontinuous (Figure 6H). Furthermore, the size and shape of the mitochondria varied greatly in the HFD-fed animals but not in the chow-fed animals (Figure 6F, white arrows).

To determine whether cellular injury (Figure 6, A-D) and autophagy (Figure 6, E-I) ultimately give way to permanent alteration of POMC neurons, we quantified POMC-immunopositive cells in long-term HFD-fed mice. This analysis revealed an approximately $25 \%$ reduction in the number of POMC cells in the ARC of mice after 8 months of exposure to HFD relative to that of controls fed chow over the same time period $(P<0.05$; Figure $6, \mathrm{~J}-\mathrm{L})$. Taken together, these results suggest that the hypothalamic response to HFD consumption involves acute injury to - and eventual loss of ARC POMC neurons.

Evidence of hypothalamic gliosis in obese humans. Based on our findings of reactive gliosis, microglial accumulation, and neuronal injury in rodents with $\mathrm{DIO}$, we investigated whether $\mathrm{MBH}$ gliosis is also associated with obesity in humans. We used magnetic resonance images obtained from a retrospective cohort of 34 subjects who had clinical MRI examinations without identified abnormalities. BMI ranged from lean to obese $\left(17.7-44.1 \mathrm{~kg} / \mathrm{m}^{2}\right)$. To determine whether visual evidence of gliosis was present in the brain images, we performed an initial inspection of the $\mathrm{MBH}$ for hyperintense signal on T2-weighted coronal sections, a characteristic finding of gliosis in numerous inflammatory, ischemic, and degenerative neural disorders (16-19). By this analysis, no studies were rated as abnormal. To detect gliotic changes below the visual detection threshold, ratios were created to compare mean signal intensity within regions of interest (ROIs) placed in the MBH with ROIs in adjacent amygdalar tissue (ROIs indicated by green circles and arrows in Figure 7, A and $\mathrm{B}$, on representative images from a lean and an obese subject). The putamen was used as a control ROI. Mean signal intensities were highly correlated between ROIs in the right and left hemispheres for all 3 brain regions (MBH: $r=0.99, P<0.0001$; amygdala: $r=0.99$, $P<0.0001$; putamen: $r=0.97, P<0.0001$ ), such that $\mathrm{MBH} /$ amygdala mean signal ratios were similar on both sides of the brain (left: $1.13 \pm 0.12$, right: $1.08 \pm 0.14 ; r=0.54, P=0.001$ ).

Across all subjects, left $\mathrm{MBH} /$ amygdala signal intensity was positively correlated with BMI, both by simple linear regression of mean intensity ratios ( $r=0.38, P=0.027$; Figure $7 C)$ and multivariate analysis using $\mathrm{MBH}$ signal as the independent variable and amygdala signal as covariate $(P<0.05$; data not shown). By comparison, the left $\mathrm{MBH} /$ amygdala mean signal ratio was not associated 

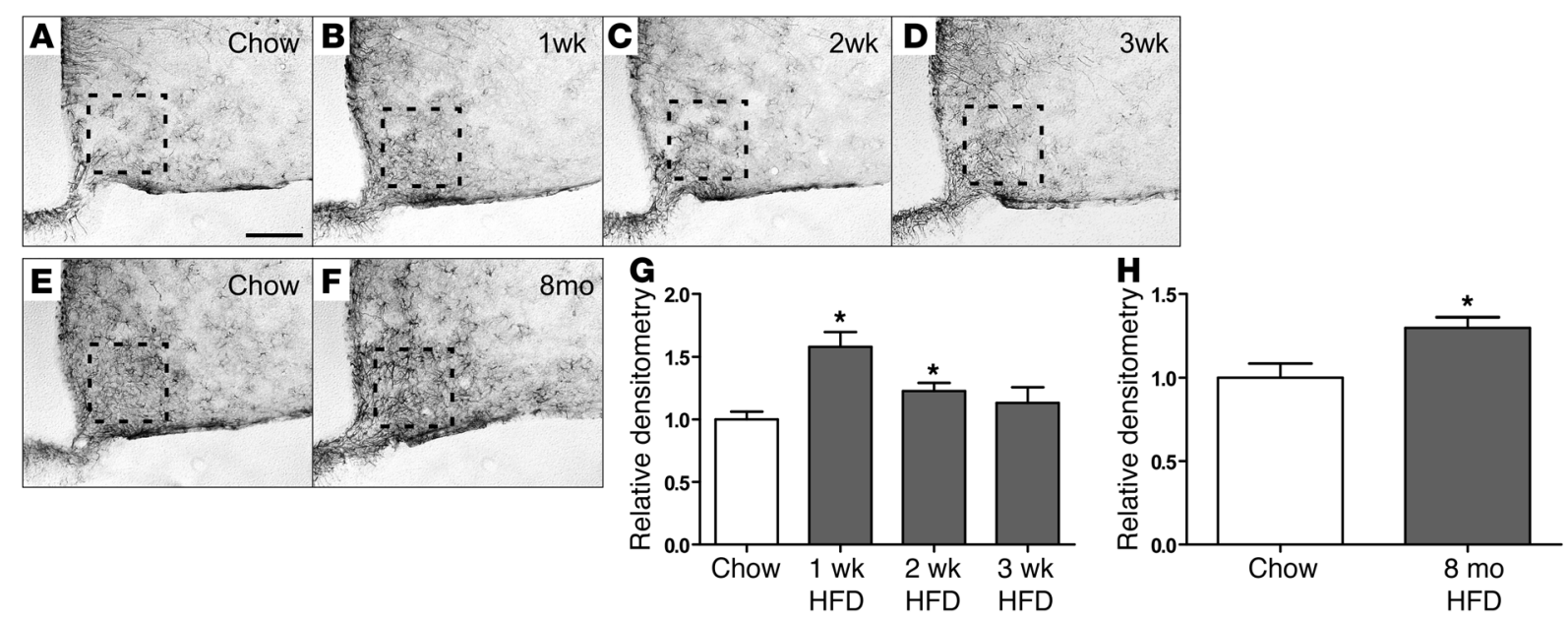

Figure 4

Time course of the effect of HFD feeding on hypothalamic astrocytes. (A-D) Representative images of astrocytes identified by immunohistochemical detection of GFAP protein in coronal sections of hypothalamus $(10 \mu \mathrm{m})$ obtained from 10-week-old mice fed either $($ A) chow or HFD for (B) 1 week, (C) 2 weeks, or (D) 3 weeks. (E and F) GFAP staining of hypothalamic sections from 8-month-old mice fed (E) chow or (F) HFD. Scale bar: $50 \mu \mathrm{m}$. (G) Quantification of GFAP staining intensity (mean \pm SEM) in the region of the ARC from mice fed either chow or HFD for 1 to 3 weeks $(n=6 /$ group). (H) Quantification of GFAP staining intensity (mean \pm SEM) in mice fed chow or HFD for 8 months. ${ }^{*} P<0.05$ versus chow. The dashed boxes indicate the region used for quantification in $\mathbf{G}$ and $\mathbf{H}$.

with age $(r=0.15 ; P=\mathrm{NS})$, and it did not differ by gender (men: $1.14 \pm 0.02$; women: $1.11 \pm 0.03 ; P=\mathrm{NS}$ ). In group comparisons, the left $\mathrm{MBH} /$ amygdala mean signal ratio was significantly higher among obese subjects $\left(1.18 \pm 0.04 ; n=12\right.$ with BMI $\left.>30 \mathrm{~kg} / \mathrm{m}^{2}\right)$ than that in normal-weight subjects $(1.06 \pm 0.04 ; n=11$ with BMI $\left.19-25 \mathrm{~kg} / \mathrm{m}^{2} ; P<0.05\right)$. Interestingly, the association of BMI with increased signal intensity appeared to be limited to the $\mathrm{MBH}$, as $\mathrm{BMI}$ was not correlated with putamen/amygdala signal ratios (left: $r=0.10, P=\mathrm{NS}$; right: $r=0.01, P=\mathrm{NS}$ ). Thus, similar to the findings in HFD-fed rodents, our retrospective analysis suggests that obesity in humans is associated with gliosis in the $\mathrm{MBH}$, raising the possibility of a common mechanism of obesity-induced hypothalamic injury across species.

\section{Discussion}

Although hypothalamic inflammation is well documented in rodent models of DIO $(7-11,20)$, neither its underlying mechanisms nor its relevance to human obesity are understood. Here we report that hypothalamic inflammation induced by HFD feeding is a manifestation of neuron injury that in turn triggers a reactive gliosis involving both microglial and astroglial cell populations. Moreover, these responses appear to occur selectively in the ARC and rapidly follow the initiation of a HFD. The transient nature of this hypothalamic response suggests that neuroprotective responses are mounted that limit or reverse the injury during its initial phases, but, with sustained exposure to the HFD, ARC-ME gliosis and injury responses are reestablished. Combined with MRI-based evidence for gliosis in the $\mathrm{MBH}$ of obese humans, our findings suggest that, in both humans and rodent DIO models, obesity is associated with neuron injury in a brain area crucial for body weight control.

Several mechanisms have been forwarded to explain obesityinduced inflammation in both peripheral tissues and hypothalamus, including activation of TLR4, induction of endoplasmic reticulum stress, and activation of serine/threonine kinases, such as IKK $\beta$ (reviewed in ref. 8). While the contribution made by these mechanisms remains uncertain, the much earlier onset of inflammation in hypothalamus relative to that in peripheral tissues raises the possibility that different processes are involved. Moreover, the nature of the hypothalamic inflammation occurring during the first days of HFD feeding may differ fundamentally from that involved with chronic HFD exposure. The conclusion that the rapid onset of $\mathrm{MBH}$ inflammation is a manifestation of neuron injury and associated neuroprotective responses is consistent with previous evidence of apoptosis and glial ensheathment of ARC neurons in animals rendered obese by chronic HFD feeding $(29,30)$. Moreover, these responses were detected specifically in ARC POMC cells $(29,30)$, which is consistent with our finding of an approximately $25 \%$ reduction in the number of hypothalamic POMC neurons in mice chronically fed a HFD. In this context, it is noteworthy that POMC cells play an essential role to protect against obesity and that loss of these cells is sufficient in and of itself to cause excess weight gain in mice (31).

In experimental models of brain injury, astrocytes play a key neuroprotective role, limiting the extent of both inflammation and neuron loss $(21,22)$. The reactive gliosis we observed in the $\mathrm{MBH}$ of both rats and mice may therefore be neuroprotective in nature, limiting local injury induced by HFD feeding. Consistent with this hypothesis is our finding that the return of proinflammatory markers in the ARC-ME area to basal, preintervention values (on day 7 of HFD feeding) coincides with the appearance of a reactive gliosis in the same brain area. Like markers of inflammation, however, this gliosis is initially transient, but both responses are reestablished as obesity develops (within 4 weeks of HFD feeding). These observations suggest that with sustained exposure the capacity of supportive glial cells to control the damage associated with HFD feeding is exceeded and that neuron injury and loss can no longer be prevented.

This hypothesis is consistent with our finding that during HFD feeding, Hsp72 was rapidly induced in neurons in the ARC-ME. Heat shock proteins are induced in response to many forms of brain injury, including stroke, neurodegenerative disease, epilep- 


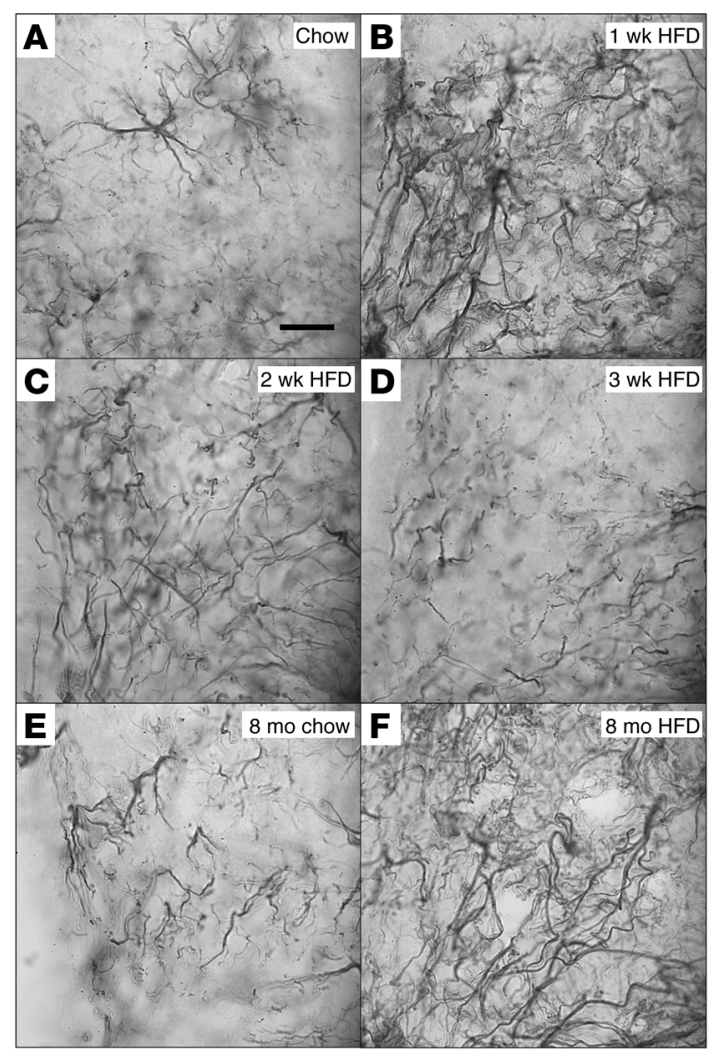

sy, and trauma, and overexpression of these proteins can serve a protective role in models of nervous system injury $(26,32)$. Hsp72 functions as a chaperone and protects neurons from protein aggregation and toxicity (in Parkinson disease, Alzheimer disease, polyglutamine diseases, and amyotrophic lateral sclerosis) as well as apoptosis (in Parkinson disease) $(26,32)$. It is also a stress marker (temporal lobe epilepsy) and protects cells from inflammation (cerebral ischemic injury) $(26,32)$. Our findings therefore suggest that rapid induction of Hsp72 in ARC neurons during HFD feeding is part of a neuroprotective response that also involves local microglial and astroglial responses.

Autophagy is a lysosomal degradative pathway that maintains cellular homeostasis by turning over cellular components. Interestingly, this process was recently suggested to participate in the physiological response of $\mathrm{MBH}$ neurons to fasting, based on evidence that fasting-induced autophagy liberates nutrient-related signals that regulate neuron firing (33). Beyond the response to starvation, however, autophagy is also a prominent feature of numerous pathological processes (e.g., neurodegeneration) and can lead to apoptosis of neurons and other cell types $(27,28,34)$. Although the precise interpretation of increased autophagy in the setting of HFD feeding awaits additional study, recent evidence suggests it may serve to minimize neuronal inflammation and injury (35). Consistent with this interpretation, the apoptosis of ARC POMC cells in rats with $\mathrm{DIO}(30)$ and the $25 \%$ reduction in POMC cell number we observed in HFD-fed mice support the hypothesis that increased numbers of autophagosomes in this cell population reflect ongoing cell injury and that this protective response along with others, such as upregulated chaperone expression and endoplasmic reticulum stress $(11,36)$, are ultimately insufficient to prevent cell loss with

\section{Figure 5}

Effect of HFD feeding on ARC astrocyte morphology. High-magnification (original magnification, $\times 100$ ) examination of astrocyte processes by GFAP immunohistochemistry of sections through mouse ARC. (A) Astrocyte processes in the ARC of mice fed chow remain separated into discrete areas. (B) One week of HFD feeding is accompanied by the apparent formation of a syncytium of astrocytic processes. (C) This astrocyte response is partially resolved by 2 weeks of HFD feeding, with only a few scattered overlapping processes, and, (D) by 3 weeks of HFD, glial morphology appears to be fully normalized. (E) Mice fed chow for 8 months show increased astrocyte number but no overlap of processes. (F) Mice fed HFD for 8 months exhibit severe astrocytosis suggestive of syncytium formation. Scale bar: $10 \mu \mathrm{m}$.

continued exposure to HFD. This model warrants further study with interventions to determine whether susceptibility to DIO is altered by manipulation of these protective responses.

Although microglia can be activated in a proinflammatory manner and cause tissue damage $(37,38)$, their diverse roles to regulate and support neuronal function are a topic of intense recent interest (37-39). For example, microglia participate in synaptic pruning both during development (39) and in adult brain $(37,38)$, and they play an important neuroprotective role under conditions in which the initial insult is directed at neurons (e.g., demyelinating or neurodegenerative disease) $(23,24)$, rather than at microglia themselves. Available evidence suggests that in the first few days of HFD feeding, the inflammation and neuron injury we have observed in the ARC-ME is not due to actions of microglia. Rather, we hypothesize a neuroprotective effect of these cells based on our findings that (a) accumulation and enlargement of microglia becomes detectable in the ARC only after inflammation is established in this brain area, and (b) this microglial response persists even as local inflammation resolves (albeit transiently). With chronic HFD feeding, however, a pathogenic role for proinflammatory microglia has not been excluded, and future studies are warranted to address this issue. An additional unanswered question is why astrocytosis in the ARC resolves temporarily (along with a decrease of proinflammatory markers) after the first week of HFD feeding, whereas microglial accumulation continues to increase during this time.

It is important to note that the current studies were undertaken in rodent strains known to be genetically predisposed to DIO, and the important question of whether the hypothalamic response to HFD feeding differs in obesity-resistant strains remains unanswered. This point is particularly germane in light of evidence that the balance between excitatory and inhibitory synaptic contacts on ARC neurons is altered by $\operatorname{HFD}$ feeding $(29,40)$ in a manner that differs between animals that are genetically predisposed to DIO and those that are obesity resistant (29). Given the capacity of both microglia and astrocytes to participate in synaptic remodeling and modulate synaptic levels of various neurotransmitters (21-24, 37, $41)$, it will be of interest in future studies to determine whether hypothalamic responses to HFD feeding are influenced by genetic factors that impact predisposition to DIO.

Based on literature establishing structural MRI as a reliable method for visualizing and quantifying gliosis in human brain (16-19), we undertook a retrospective analysis of T2-weighted magnetic resonance images obtained previously in a cohort of young human subjects undergoing clinical examination. We found hyperintensity of the T2-weighted signal in human $\mathrm{MBH}$ that was significantly increased $(P<0.05)$ in obese individuals compared with that in 

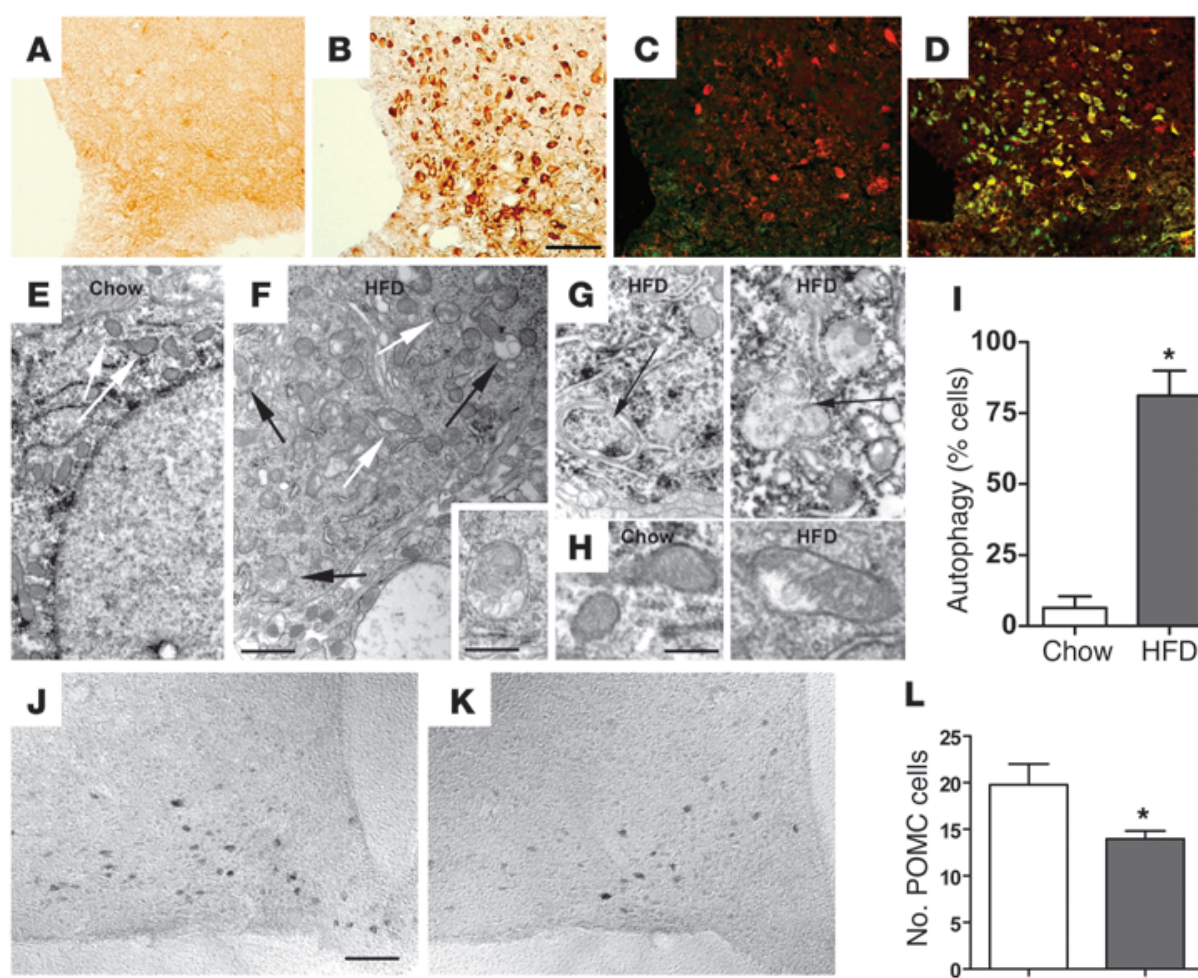

I

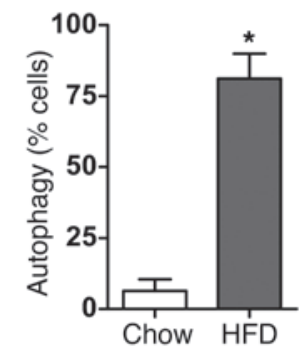

$\mathbf{L}$

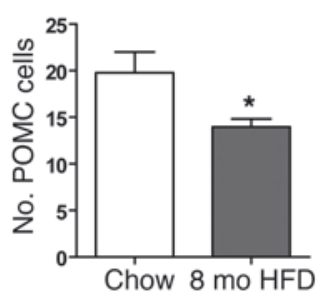

\section{Figure 6}

Effect of HFD feeding on hypothalamic markers of neuronal injury and on POMC cell number. (A-D) Immunohistochemical analysis of the neuronal injury marker Hsp72 in the ARC of rats fed (A and C) chow or (B and $\mathbf{D}$ ) HFD for 7 days. Immunofluorescence in $\mathbf{C}$ and $\mathbf{D}$ shows colocalization of Hsp72 (green) with POMC peptide (red). (E and F) Electron micrograph of a POMC neuron from a mouse fed (E) chow or (F) HFD for 20 weeks. In E, no pathological changes in cytoplasm or mitochondria (white arrows) are observed, whereas, in $\mathbf{F}$, mitochondrial integrity is disrupted (white arrows), and mature and developing autophagosomes are present (black arrows). The inset in $\mathbf{F}$ is a higher-magnification example of an autophagosome. (G) Additional high-magnification examples of autophagosomes (black arrows). (H) High-magnification images of POMC neuron mitochondria from mice fed chow (parallel-oriented cristae and regular structure) or HFD (nonparallel cristae and irregular shape). (I) Quantification of percentage of POMC neurons with autophagosomes ( $n=5$ cells examined in each of 5 mice). ${ }^{*} P<0.05$ versus chow. ( $\mathbf{J}$ and $\mathbf{K}$ ) Representative images of POMC neurons in the ARC of mice fed either (J) chow or (K) HFD for 8 months. (L) Quantification of POMC neuron number in the hypothalamus of mice fed chow or HFD for 8 months (mean $\pm \mathrm{SEM} ; n=8$ /group). ${ }^{\star} P<0.05$ versus chow. Scale bar: $50 \mu \mathrm{m}(\mathbf{A}-\mathbf{D}, \mathbf{J}$, and $\mathbf{K}) ; 1 \mu \mathrm{m}$ (E and $\mathbf{F}) ; 500 \mathrm{~nm}(\mathbf{F}$, inset); $400 \mathrm{~nm}(\mathbf{G}$ and $\mathbf{H})$.

lean individuals, indicative of gliosis. This finding does not constitute definitive proof of increased gliosis, as edema, infection, and tumors can have a similar appearance. However, subjects having preexisting evidence of neurological abnormality were excluded from the study, suggesting that these alternative explanations for increased T2-weighted signal intensity are unlikely. Furthermore, as predicted by our findings in rodents, the gliosis signal was positively correlated to BMI but not to age or gender. Although the magnitude of this increase associated with obesity was modest (which likely reflects, at least in part, limitations inherent in gliosis detection by MRI), these data lend translational relevance to our preclinical studies by suggesting that hypothalamic neuron injury and associated gliosis are a feature of obesity in humans as well as rodents. Although it remains to be determined whether this finding is causally linked to obesity pathogenesis or is simply a marker of the obese state, it seems reasonable to anticipate that damage to a critical brain area for body weight control might play a role in the associated obesity (42), and studies to clarify this issue are a high priority for future research.

In summary, we report that, in rats and mice that are susceptible to DIO, consumption of a HFD rapidly induces neuron injury in a brain area critical for energy homeostasis. Although local responses appear to limit this injury, recovery is transient, eventually giving way to chronic inflammation, neuron loss, and reactive gliosis. Extending these findings is MRI evidence for gliosis in the hypothalamus of obese humans. Collectively, this work identifies a potential link between obesity and hypothalamic injury in humans as well as animal models.

\section{Methods}

Animals. Weight-matched male LongEvans rats (300-350 g; Harlan) or male C57BL/6 mice (20-25 g) were housed individually in a specific pathogen-free environment, maintained in a temperature-controlled room with a 12 -hourlight/12-hour-dark cycle, and provided with ad libitum access to water and either standard laboratory chow $(3.34 \mathrm{kcal} / \mathrm{g}$; PMI Nutrition International) or a diet containing $60 \% \mathrm{kcal}$ fat (HFD, $5.24 \mathrm{kcal} / \mathrm{g}$, D12492; Research Diets) for periods ranging from 1 day to 8 months. Body weight and food intake were monitored daily.

Real-time PCR. For expression analyses, RNA from liver, epididymal white adipose tissue, and $\mathrm{MBH}$ (rectangular block excised as previously described; ref. 43) was extracted using TRIzol B according to the manufacturers' instructions (MRC). RNA was quantified by spectrophotometry at $260 \mathrm{~nm}$ (Nanodrop 1000; Thermo Scientific) and reverse transcribed with avian myeloblastosis virus reverse transcriptase ( $1 \mu \mathrm{g}$; Promega). Levels of mRNA for Nfkbia, Ikbkb, Ikbke, Il6, Il1b, Tnfa, Gfap, Emr1, Cd68, Gapdh (internal control), and $18 S$ RNA (internal control) were measured by semiquantitative real-time PCR on an ABI Prism 7900 HT (Applied Biosystems). The primer sequences were designed using Primer Express (version 2.0.0; Applied Biosystems) as follows: Nfkbia, forward, TGCCTGGCCAGTGTAGCAGTCTT, reverse, CAAAGTCACCAAGTGCTCCACGAT; rat Ikbkb, forward, AGGGTGACTAAGTCGAGAC, reverse, ACAGCCAGGATATGGTACG; mouse Ikbkb, forward, GGCACCTTGGATGACCTAGA, reverse, CCATATCCTGGCTGTCACCT; Ikbke, forward, ACCACTAACTACCTGTGGCAT, reverse, ACTGCGAATAGCTTCACGATG; rat Il6, forward, CAGAGGATACCACCCACAACAGA, reverse, CAGTGCATCATCGCTGTTCATACA; mouse $I l 6$, forward, GTGGCTAAGGACCAAGACCA, reverse, GGTTTGCCGAGTAGACCTCA; $I l 1 b$, forward, TACAAGGAGAGACAAGCAACGACA, reverse, GATCCACACTCTCCAGCTGCA; rat Tnfa, forward, GCTCCСТCTCATCAGTTCCA, reverse, CTCCTCTGCTTGGTGGTTTG; mouse Tnfa, 

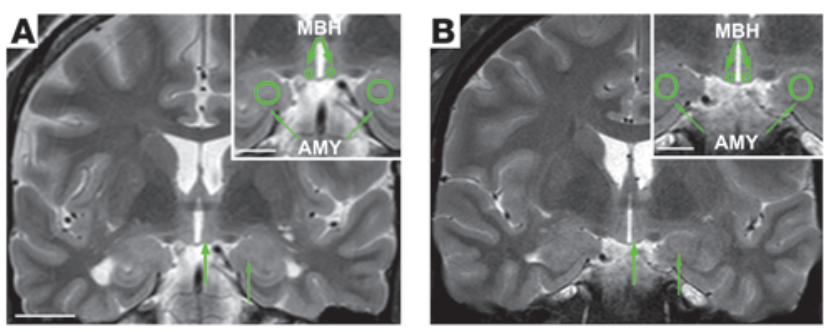

C

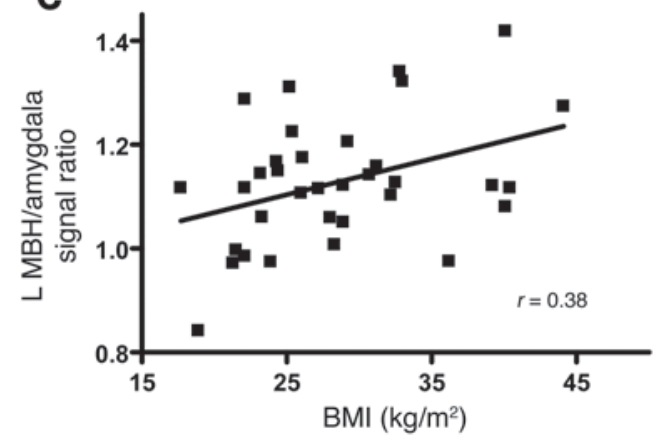

forward, CATCTTCTCAAAACTCGAGTGACAA, reverse, TGGGAGTAGATAAGGTACAGCCC; Gfap, forward, AACGACTATCGCCGCCAACTG, reverse, CTCTTCCTGTTCGCGCATTTG; rat Emr1, forward, AATCGCTGCTGGCTGAATACGG, reverse, CCAGGCAAGGAGGGCAGAGTT; mouse Emr1, forward, AATCGCTGCTGGTTGAATACAG, reverse, CCAGGCAAGGAGGACAGAGTT; Cd68, forward, CTTCCCACAAGCAGCACAG, reverse, AATGATGAGAGGCAGCAAGAGA; Gapdh, forward, AACGACCCCTTCATTGAC, reverse, TCCACGACATACTCAGCAC; and 18S forward, CGGACAGGATTGACAGATTG, reverse, CAAATCGCTCCACCAACTAA. PCR data were analyzed using the Sequence Detection System software (SDS version 2.2; Applied Biosystems). Expression levels of each gene were normalized to housekeeping genes (Gapdh and 18S) and expressed as a percentage of chow-fed controls. Nontemplate controls were incorporated into each PCR run.

Immunohistochemistry. After fixation-perfusion with $4 \%$ paraformaldehyde/ PBS, frozen sections in the coronal plane through the rat and mouse hypothalamus were processed for GFAP, Iba1, POMC, and Hsp72 immunoreactivity using standard immunohistochemical procedures. Sections blocked in 5\% normal goat, mouse, or donkey serum (Jackson ImmunoResearch Laboratories Inc.) were incubated overnight at $4^{\circ} \mathrm{C}$ with mouse anti-GFAP $(1: 10,000$; Sigma-Aldrich), mouse anti-Hsp72 (1:1,000; Enzo Life Sciences), rabbit antiIba1 (1:1,000; Wako Pure Chemicals), goat anti-Iba1/AIF-1 (1:1,000; Everest Biotech), and anti-POMC (Ab5, 1:10,000; AbCam). Biotinylated anti-rabbit, anti-mouse or anti-goat secondary antibody, $\mathrm{ABC}$ reagent, and diaminobenzidine substrate (Vector Laboratories) were used for light microscopic visualization. Immunofluorescence was performed with a combination of Alexa Fluor 488- or Alexa Fluor 594-labeled anti-rabbit, anti-goat, or antimouse secondary antibodies (1:500; Invitrogen) and DAPI (Sigma-Aldrich) to identify cell nuclei. GFAP and Iba1 antibodies have been widely validated in the literature as markers for astrocytes and microglia, respectively. In addition, we saw no overlap between these markers and NeuN, a neuronal marker (data not shown). The POMC antibody identified only a small population of neurons in the expected area of the ARC (Figure 6, C, D, J, and K) and hindbrain (data not shown), consistent with the known location of these cells. Hsp72 immunostaining has been used extensively in prior studies $(26,32)$. In addition, sections incubated with preimmune serum showed no detectable signal (data not shown). While the absence of detectable immunoreac-

\section{Figure 7}

Radiologic evidence of gliosis in the MBH of obese humans. Representative coronal T2-weighted images through the hypothalamus from (A) a normal weight and (B) an obese subject. Insets show the placement of right and left ROIs (green circles) in the $\mathrm{MBH}$ and amygdala (AMY). In the MBH (thick arrows) of the obese subject, signal ratios demonstrated subtle hyperintensity (brightness) relative to the amygdala (thin arrows). Scale bar: $20 \mathrm{~mm} ; 10 \mathrm{~mm}$ (insets). (C) Correlation of $\mathrm{BMI}$ with $\mathrm{MBH}$ hyperintensity as measured by left $(\mathrm{L}) \mathrm{MBH} / \mathrm{amygdala}$ signal ratio ( $n=34$ subjects; $r=0.38 ; P=0.027$ ).

tivity in chow-fed controls and presence of positive staining in HFD brains indicates that the Hsp72 antibody staining is not nonspecific, we cannot absolutely rule out the possibility that the antibody may recognize other antigens in hypothalamic neurons of HFD-fed rats.

Images were captured on an Eclipse E600 upright microscope equipped with a color digital camera (Nikon). Quantification was performed in a blinded fashion on anatomically matched brain regions identified in $\times 20$ images. Both sides of bilateral structures (e.g., ARC, VMH, etc.) were counted on 2 to 4 slides per animal, and replicate values from each animal were individually averaged before determining group means ( $n=4-8$ /group). For Iba1 and POMC immunostaining in which discrete cells could be identified, cell number was counted manually within prespecified ROIs using Photoshop (Adobe) or ImageJ (http://rsbweb.nih. gov/ij/). Signal intensity was scored in a semiquantitative fashion and was found to covary with diet group. For microglial cell size (using Iba1) and GFAP immunoreactivity, thresholding was performed in ImageJ, followed by densitometric quantification.

Autophagy analysis. Group-housed C57BL/6 mice fed either standard chow (Purina Lab Chow no. 5001, Ralston Purina Corp.) or 45\% kcal HFD (D12451, Research Diets) for 20 weeks were perfused with picric acid, and their brains were processed for electron microscopic examination. Ultrathin sections were cut on a Leica ultramicrotome, collected on Formvar-coated single-slot grids, and analyzed with a Tecnai 12 Biotwin electron microscope (FEI Company). The analysis of autophagosome (intracellular membranebound cytosol and organelles) number was performed in an unbiased fashion, as described elsewhere $(29,44)$. We analyzed 5 POMC neurons identified by POMC immunolabeling in 5 animals per experimental group.

Retrospective study of brain MRIs in humans. We performed a retrospective cohort study to assess for radiologic evidence of $\mathrm{MBH}$ gliosis and correlated our findings with BMI levels and obesity. Magnetic resonance brain examinations, using pituitary or epilepsy imaging protocols that used coronal T2-weighted sequences performed between 1/1/2009 and $12 / 31 / 2010$ at University of Washington Medical Center or Harborview Medical Center, were reviewed for 2 inclusion criteria: (a) availability of good quality coronal views of the hypothalamus and (b) absence of clinical abnormalities that might confound interpretation (e.g., hypothalamic-pituitary axis disease). For 39 studies that met our inclusion criteria, we reviewed electronic medical records to determine body weight at time of exam, height, age, gender, indication for magnetic resonance examination, final diagnosis, comorbidities (e.g., diabetes mellitus), and maximum recorded BMI. Exclusion criteria were as follows: BMI not available; age of less than 18 or more than 70 years; diagnosis of pituitary, hypothalamic, or neurodegenerative disorder (e.g., multiple sclerosis); cerebral atrophy; or history of bariatric surgery. Five subjects were excluded (age > $70[n=2]$; missing height $[n=1]$; type 1 diabetes mellitus $[n=1]$; CNS disease $[n=1])$, yielding a total of 34 study participants (19 men, 15 women; mean age, $38 \pm 12$ years of age [range $18-63$ years of age]; mean BMI, $28.5 \pm 6.7 \mathrm{~kg} / \mathrm{m}^{2}$ [range $\left.17.7-44.1 \mathrm{~kg} / \mathrm{m}^{2}\right]$ ). The most common indications for examination were hypogonadism (29\%), elevated prolac- 
tin levels (21\%), and seizure (15\%). On targeted visual inspection of the $\mathrm{MBH}$, no studies were rated as abnormal, 7 were rated as equivocal (4 left, 1 right, 2 bilaterally), and 27 were rated were normal. There was no difference in the proportion of normal weight versus obese subjects who had an equivocal finding (Fisher's exact $=1.000 ; P=\mathrm{NS}$ ).

Single coronal slices through the hypothalamus, using T2-weighted fast spin echo (with or without fat saturation) or fluid-attenuated inversion recovery sequences, were identified for each subject. The $\mathrm{MBH}$ was visually inspected for abnormalities and rated as normal, equivocal, or abnormal. ROIs in the right and left $\mathrm{MBH}$, the right and left amygdala, and the right and left putamen were defined by a neuroradiologist, who was blinded to all clinical information. Mean ROI signal intensity, standard deviation, and area of ROI were measured by using proprietary software on the PACS workstation (Centricity, GE Healthcare). Ratios were calculated by comparing the mean signal intensity in the $\mathrm{MBH}$ on each side with that in the ipsilateral amygdala ROI. A control ratio compared mean signal intensity in the putamen with that in the ipsilateral amygdala. Subjects were classified into 2 groups: obese (BMI $>30 \mathrm{~kg} / \mathrm{m}^{2}$ ) and normal weight (BMI $19-24.9 \mathrm{~kg} / \mathrm{m}^{2}$, without any previously recorded BMI $>30$ ). Subjects with BMIs outside these ranges were excluded from group analyses, but data from all subjects were used in correlation analyses.

Statistics. All results are expressed as mean \pm SEM. Statistical analyses were performed using GraphPad PRISM (version 4.0b; Graph Pad Software). One-way ANOVA with Dunnet's least significant difference posthoc tests was used to compare mean values against those for chow controls, while 2-tailed Student's $t$ tests were used for 2-group comparisons. For the human MRI study, group differences were assessed by unpaired 2-tailed Student's $t$ test for continuous variables and Fisher's exact test for categorical variables. Normal distributions were confirmed for continuous variables. Pearson's correlation coefficients were calculated, and univariate linear regression was used to test for significance. A secondary analysis was performed as an alternate method to using signal ratios by using mean $\mathrm{MBH}$ signal intensity in a multivariate regression, with BMI as the independent variable and mean amygdala signal intensity as a covariate (performed in Stata 9.2, Statacorp). In all instances throughout, $P$ values of less than 0.05 were considered significant.

Study approval. All study protocols involving rats and mice were approved by the Animal Care and Use Committees at the University of Washington, Uni- versity of Cincinnati, or Yale University and conducted in accordance with the $\mathrm{NIH}$ guidelines for care and use of animals. The study involving analysis of human MRI scans was approved by the University of Washington Institutional Review Board (IRB). The IRB granted a waiver of informed consent; because this was a retrospective study that involved minimal risk to the involved subjects, it was not possible to contact all of the subjects, and appropriate safeguards were in place to protect subject confidentiality and privacy.

\section{Acknowledgments}

We thank A. Cubelo, L. Nguyen, C. Davis, I. David, and K. Ogimoto for technical assistance with the studies described in this manuscript. This work was supported by a NIH Career Development Award (DK088872) and Diabetes Endocrinology Research Center (DERC) Pilot and Feasibility Award (DK017047) to J. Thaler; NIH grants to M. Schwartz (DK068384, DK083042 and DK052989), M. Tschöp (DK077975), and T. Horvath (DK080000); The Netherlands Organization for Scientific Research - ALW Rubicon to C. Yi; and the Merit Review Research and Research Enhancement Award Programs of the Office of Research and Development, Department of Veterans Affairs to D. Baskin. D. Baskin is the recipient of a Department of Veterans Affairs Senior Research Career Scientist Award. Additional assistance and support was provided by the Nutrition Obesity Research Center (DK035816), Mouse Metabolic Phenotyping Center (U24 DK076126), and Cellular and Molecular Imaging Core of the DERC (DK017047) at the University of Washington.

Received for publication September 30, 2011, and accepted in revised form November 2, 2011.

Address correspondence to: Michael Schwartz, Diabetes and Obesity Center of Excellence, University of Washington School of Medicine, 815 Mercer St., Box 358055, Seattle, Washington 98109, USA. Phone: 206.897.5288; Fax: 206.897.5293; E-mail: mschwart@ u.washington.edu.

David A. Sarruf's present address is: Diabetes Pharmacology, Novo Nordisk A/S, Maaloev, Denmark.
1. Morton GJ, Cummings DE, Baskin DG, Barsh GS, Schwartz MW. Central nervous system control of food intake and body weight. Nature. 2006; 443(7109):289-295.

2. Myers MG, Cowley MA, Munzberg H. Mechanisms of leptin action and leptin resistance. Annu Rev Physiol. 2008;70:537-556.

3. Myers MG Jr, Leibel RL, Seeley RJ, Schwartz MW. Obesity and leptin resistance: distinguishing cause from effect. Trends Endocrinol Metab. 2010; 21(11):643-651.

4. Lumeng CN, Saltiel AR. Inflammatory links between obesity and metabolic disease. J Clin Invest. 2011;121(6):2111-2117.

5. Schenk S, Saberi M, Olefsky JM. Insulin sensitivity: modulation by nutrients and inflammation. J Clin Invest. 2008;118(9):2992-3002.

6. Shoelson SE, Lee J, Goldfine AB. Inflammation and insulin resistance. $J$ Clin Invest. 2006; 116(7):1793-1801.

7. De Souza CT, et al. Consumption of a fat-rich diet activates a proinflammatory response and induces insulin resistance in the hypothalamus. Endocrinology. 2005;146(10):4192-4199.

8. Thaler JP, Schwartz MW. Minireview: Inflammation and obesity pathogenesis: the hypothalamus heats up. Endocrinology. 2010;151(9):4109-4115.
9. Kleinridders A, et al. MyD88 signaling in the CNS is required for development of fatty acid-induced leptin resistance and diet-induced obesity. Cell Metab. 2009;10(4):249-259.

10. Milanski M, et al. Saturated fatty acids produce an inflammatory response predominantly through the activation of TLR4 signaling in hypothalamus: implications for the pathogenesis of obesity. J Neurosci. 2009;29(2):359-370.

11. Zhang X, Zhang G, Zhang H, Karin M, Bai H, Cai D. Hypothalamic IKKbeta/NF-kappaB and ER stress link overnutrition to energy imbalance and obesity. Cell. 2008;135(1):61-73.

12. Posey KA, et al. Hypothalamic proinflammatory lipid accumulation, inflammation, and insulin resistance in rats fed a high-fat diet. Am J Physiol Endocrinol Metab. 2009;296(5):E1003-E1012.

13. Kim F, et al. Vascular inflammation, insulin resistance, and reduced nitric oxide production precede the onset of peripheral insulin resistance. Arterioscler Thromb Vasc Biol. 2008;28(11):1982-1988.

14. Mori MA, et al. A systems biology approach identifies inflammatory abnormalities between mouse strains prior to development of metabolic disease. Diabetes. 2010;59(11):2960-2971.

15. Weisberg SP, McCann D, Desai M, Rosenbaum M, Leibel RL, Ferrante AW Jr. Obesity is associated with macrophage accumulation in adipose tissue. J Clin Invest. 2003;112(12):1796-1808.

16. Braffman BH, Zimmerman RA, Trojanowski JQ, Gonatas NK, Hickey WF, Schlaepfer WW. Brain MR: pathologic correlation with gross and histopathology. 2. Hyperintense white-matter foci in the elderly. AJR Am J Roentgenol. 1988;151(3):559-566.

17. Briellmann RS, Kalnins RM, Berkovic SF, Jackson GD. Hippocampal pathology in refractory temporal lobe epilepsy: T2-weighted signal change reflects dentate gliosis. Neurology . 2002;58(2):265-271.

18. Chung YL, et al. Conflicting MRI signals from gliosis and neuronal vacuolation in prion diseases. Neuroreport. 1999;10(17):3471-3477.

19. Marshall VG, Bradley WG Jr, Marshall CE, Bhoopat $\mathrm{T}$, Rhodes RH. Deep white matter infarction: correlation of MR imaging and histopathologic findings. Radiology. 1988;167(2):517-522.

20. Posey K, et al. Hypothalamic proinflammatory lipid accumulation, inflammation, and insulin resistance in rats fed a high-fat diet. Am J Physiol Endocrinol Metab. 2009;296(5):E1003-E1012.

21. Sofroniew MV. Molecular dissection of reactive astrogliosis and glial scar formation. Trends Neurosci. 2009;32(12):638-647.

22. Pekny M, Nilsson M. Astrocyte activation and reactive gliosis. Glia. 2005;50(4):427-434. 
23. Hanisch UK, Kettenmann H. Microglia: active sensor and versatile effector cells in the normal and pathologic brain. Nat Neurosci. 2007; 10(11):1387-1394

24. Ransohoff RM, Perry VH. Microglial physiology: unique stimuli, specialized responses. Annu Rev Immunol. 2009;27:119-145.

25. Ito D, Imai Y, Ohsawa K, Nakajima K, Fukuuchi Y, Kohsaka S. Microglia-specific localisation of a novel calcium binding protein, Iba1. Brain Res Mol Brain Res. 1998;57(1):1-9.

26. Sharp FR, Massa SM, Swanson RA. Heat-shock protein protection. Trends Neurosci. 1999;22(3):97-99.

27. Chu CT. Autophagic stress in neuronal injury and disease. J Neuropathol Exp Neurol. 2006;65(5):423-432.

28. Wong E, Cuervo AM. Autophagy gone awry in neurodegenerative diseases. Nat Neurosci. 2010; 13(7):805-811.

29. Horvath TL, et al. Synaptic input organization of the melanocortin system predicts diet-induced hypothalamic reactive gliosis and obesity. Proc Natl Acad Sci U S A. 2010;107(33):14875-14880.

30. Moraes JC, et al. High-fat diet induces apoptosis of hypothalamic neurons. PLoS One. 2009;4(4):e5045. 31. Gropp E, et al. Agouti-related peptide-expressing neurons are mandatory for feeding. Nat Neurosci. 2005;8(10):1289-1291.

32. Turturici G, Sconzo G, Geraci F. Hsp70 and its molecular role in nervous system diseases. Biochem Res Int. 2011;2011:618127.

33. Kaushik S, et al. Autophagy in hypothalamic AgRP neurons regulates food intake and energy balance. Cell Metab. 2011;14(2):173-183.

34. Alirezaei M, Kemball CC, Whitton JL. Autophagy, inflammation and neurodegenerative disease. Eur J Neurosci. 2011;33(2):197-204.

35. Meng Q, Cai D. Defective hypothalamic autophagy directs the central pathogenesis of obesity via the IkappaB kinase beta (IKKbeta)/NF-kappaB pathway. J Biol Chem. 2011;286(37):32324-32332.

36. Ozcan L, et al. Endoplasmic reticulum stress plays a central role in development of leptin resistance. Cell Metab. 2009;9(1):35-51.

37. Graeber MB. Changing face of microglia. Science 2010;330(6005):783-788.

38. Dheen ST, Kaur C, Ling EA. Microglial activation and its implications in the brain diseases. Curr Med Chem. 2007;14(11):1189-1197.

39. Paolicelli RC, et al. Synaptic pruning by microglia is necessary for normal brain development. Science. 2011;333(6048):1456-1458

40. Ravussin Y, et al. Effects of chronic weight perturbation on energy homeostasis and brain structure in mice. Am J Physiol Regul Integr Comp Physiol. 2011; 300(6):R1352-R1362.

41. Perea G, Navarrete M, Araque A. Tripartite synapses: astrocytes process and control synaptic information. Trends Neurosci. 2009;32(8):421-431.

42. Hochberg I, Hochberg Z. Expanding the definition of hypothalamic obesity. Obes Rev. 2010; 11(10):709-721.

43. Thaler JP, et al. Atypical protein kinase C activity in the hypothalamus is required for lipopolysaccharidemediated sickness responses. Endocrinology. 2009; 150(12):5362-5372.

44. Dietrich MO, et al. Agrp neurons mediate Sirt1's action on the melanocortin system and energy balance: roles for Sirt 1 in neuronal firing and synaptic plasticity. J Neurosci. 2010;30(35):11815-11825. 